Conclusion: Children with JIA have adiposity similar to healthy controls. Inflammatory activity measured by JDAS is associated with fat mass but not to anthropometric measurements such as body mass index (BMI).

REFERENCES

[1] Grönlund et al Juvenile idiopathic arthritis patients with low inflammatory activity have increased adiposity. Scand J Rheumatol 2014

[2] Giani et al. The Influence of Overweight and Obesity on Treatment Response in Juvenile Idiopathic Arthritis. Front Pharmacol 2019

[3] Wiech et al. Body composition and phase angle as an indicator of nutritional status in children with juvenile idiopathic arthritis. Pediatric Rheumatology 2018

[4] Alvarez-Nemegyei et al. Association between Overweight/Obesity and Clinical Activity in rheumatoid arthritis. Reumatol Clin 2020

Disclosure of Interests: None declared

DOI: 10.1136/annrheumdis-2021-eular.3750

\section{POS1329 A COHORT STUDY ON THE RISK OF DEVELOPING OBSTRUCTIVE SLEEP APNEA, TEMPOROMANDIBULAR JOINT DISORDERS AND CRANIOFACIAL DEFORMITIES IN PATIENTS WITH JUVENILE IDIOPATHIC ARTHRITIS}

K. S. K. MA ${ }^{1}$, L. T. Wang ${ }^{2} .{ }^{1}$ National Taiwan University, Taipei, Taiwan, ROC, Department of Life Science, Taipei, Taiwan, Republic of China; ${ }^{2}$ National Taiwan University Hospital, Department of Obstetrics \& Gynecology, Taipei, Taiwan, Republic of China

Background: Juvenile Idiopathic Arthritis (JIA), an autoimmune disease, has been proposed to be comorbid with obstructive sleep apnea (OSA)

Objectives: We aimed at identifying the relationship between JIA and OSA.

Methods: We performed a cohort study including JIA and OSA patients from 1999 to 2013. A total of 2791 patients diagnosed with OSA after JIA onset were recruited, which 11,164 eligible individuals without JIA history were selected as matched-controls. A Cox proportional hazard model was developed to estimate the risk of OSA in JIA patients. A cumulative probability model was adopted to assess the time-dependent effect of JIA on OSA development, implying the casual link of the association. To identify whether JIA patients have higher risks for developing temporomandibular joint (TMJ) disorders, craniofacial anomalies and deformities than non-JIA individuals, subgroup analyses was conducted. Finally, Ingenuity Systems Pathway Analysis (IPA) was conducted to identify underlying mechanisms of the above disease correlation among peripheral blood mononuclear cells (PBMCs) from rheumatic factor (RF)-positive and RF-negative JIA patients, and subcutaneous fat tissues from OSA patients, using p-value visualization for RNA-seq analyses.

Results: The Cox proportional hazard model showed that JIA patients were more likely to have OSA than non-JIA individuals (adjusted hazard ratio $=1.949,95 \%$ $\mathrm{Cl}=1.264-3.005)$. The incidence of developing OSA was particularly high among patients who developed $\mathrm{JIA}$ aged $18-30$ years old $(\mathrm{aHR}=2.034,95 \% \mathrm{Cl}=1.305$ 3.169) and males ( $\mathrm{aHR}=1.82,95 \% \mathrm{Cl}=1.121-2.954)$. The risk of developing OSA increased within 0-36 months $(\mathrm{aHR}=2.216,95 \% \mathrm{Cl}=1.001-4.907)$ and over 60 months $(\mathrm{aHR}=2.558,95 \% \mathrm{Cl}=1.346-4.860)$ of follow-up duration after JIA onset. Subgroup analyses showed that JIA patients were more likely to have TMJ disorders (relative risk $=2.047,95 \% \mathrm{Cl}=1.446-2.898$ ) and to receive treatment for craniofacial deformities ( $R R=1.722,95 \% \mathrm{Cl}=1.38-2.148)$ than non-JIA controls. IPA analyses suggested that the underlying mechanisms involved activation of antigen presentation pathway followed by antigen presentation to CD4+ and CD8+ T lymphocytes, as well as B cell development.

Conclusion: Our findings identified high risks of developing OSA, TMJ disorders, and craniofacial deformities following JIA onset, which the underlying mechanisms may involve both cellular and humoral immunity.

Disclosure of Interests: None declared

DOI: 10.1136/annrheumdis-2021-eular.3961

\section{POS1330 \\ SAFETY OF ANAKINRA IN PATIENTS WITH CRYOPYRIN ASSOCIATED PERIODIC SYNDROMES (CAPS) USING A GRADUATED PRE-FILLED SYRINGE}

R. Papa ${ }^{1,2}$, G. Giancane ${ }^{2,3}$, H. J. Lachmann ${ }^{4}$, P. Brogan ${ }^{5}$, E. Legger ${ }^{6}$, D. Lindqvist ${ }^{7}$, S. Cederholm ${ }^{7}$, F. Bagnasco ${ }^{8}$, N. Ruperto ${ }^{9}$, M. Gattorno on behalf of Paediatric Rheumatology International Trials Organisation (PRINTO) and Eurofever registry. ${ }^{1}$ IRCCS Istituto Giannina Gaslini, UOSD Centro Malattie Autoinfiammatorie e Immunodeficienze, Genoa, Italy; ${ }^{2}$ Università degli Studi di Genova, Dipartimento di Neuroscienze, Riabilitazione, Oftalmologia, Genetica e Scienze Materno-Infantili (DiNOGMI), Genoa, Italy; ${ }^{3}$ IRCCS Istituto Giannina Gaslini, Clinica Pediatrica e Reumatologia, Genoa, Italy; ${ }^{4}$ Royal Free Campus, National Amyloidosis Centre, London, United Kingdom; ${ }^{5} \mathrm{UCL}$ Institute of Child Health, Great Ormond Street Hospital, Infection, Inflammation and Rheumatology Unit, London, United Kingdom; ${ }^{6}$ Beatrix Kinderkliniek,
University Medical Center, Department of Pediatric Rheumatology, Groningen, Netherlands; ${ }^{7}$ Sobi, Stockholm, Sweden; ${ }^{8}$ IRCCS Istituto Giannina Gaslini, Servizio di Epidemiologia e Biostatistica, Genoa, Italy; ${ }^{9}$ IRCCS Istituto Giannina Gaslini, Clinica Pediatrica e Reumatologia, PRINTO, Genoa, Italy

Background: Cryopyrin associated periodic syndromes (CAPS) are a group of ultra-rare autoinflammatory diseases caused by mutations in the NLRP3 gene, leading to overproduction of IL-1 $\beta$. CAPS includes the following subdiagnoses: familial cold autoinflammatory syndrome (FCAS), Muckle-Wells syndrome (MWS) and chronic infantile neurological cutaneous articular syndrome/neonatal-onset multisystem inflammatory disease (CINCA/NOMID), where FCAS is the mildest form of CAPS and CINCA/NOMID the most severe. A graduated pre-filled syringe of the IL-1 receptor antagonist anakinra was introduced to meet the need of smaller and varying doses when treating children with CAPS.

Objectives: To evaluate the safety of anakinra in CAPS patients using the nove pre-filled syringe.

Methods: Investigators managing patients with CAPS treated with anakinra were identified via the Eurofever registry. Follow-up data of at least 3 years for each patient were prospectively collected and analyzed (EUPAS6366). The primary study endpoints were the occurrence of adverse events (AEs) with focus on serious infections, malignancies, injection site reactions, allergic reactions and medication errors, including re-use of the syringe. Secondary endpoints included dose at specific time points, discontinuations, and switch to other IL-1 blocking treatment.

Results: 12 patients with CAPS were included in the study, 8 with MWS, 2 with FCAS and 2 with CINCA/NOMID. The majority were male $(75 \%)$ and white $(83.3 \%)$. At baseline, all but one patient were already using the graduated prefilled syringe with anakinra. 5 patients required a median dose of 2 to $3 \mathrm{mg} / \mathrm{kg} /$ day and 6 patients below $2 \mathrm{mg} / \mathrm{kg} /$ day. During a total of 26.1 patient years of treatment, there were $7 \mathrm{AEs}$ with rate of $26.8(95 \% \mathrm{Cl} 4.2-169.6)$ per 100 patient years. All AEs were infections from 1 patient with CINCA/NOMID and were considered unrelated to anakinra by the investigator. Two of the AEs were considered serious as hospitalization was required ( 1 tonsillitis and 1 urinary tract infection). No AEs were severe, 6 of the AEs were of moderate severity and 1 was of mild severity. In total, 6 patients discontinued anakinra permanently, 2 during the first year of treatment, 3 during the second year and 1 after the third year of follow-up. The reasons for discontinuation included switch to canakinumab (4 patients), switch to canakinumab and inefficacy (1 patient) and non-compliance (1 patient). The remaining 5 patients continued anakira until the end of the study. 1 patient discontinued anakinra temporarily during the second year of treatment due to non-compliance. No deaths, malignancies, injection site reactions, allergic reactions or medication errors, including re-use of the pre-filled syringe, were observed.

Table 1. Study results

\begin{tabular}{lc}
\hline Patients & $12(100)$ \\
Gender, M:F & $9: 3(75: 25)$ \\
Ethnicity, white:black & $10: 2(83.3: 16.7)$ \\
Age at baseline, median (range), years & $25.3(1.4-54.9)$ \\
Age at disease onset, median (range), years & $2.5(0-29.5)$ \\
Age at diagnosis, median (range), years & $24.4(0.6-54.6)$ \\
Disease duration, median (range), years & $15.5(1.4-51.3)$ \\
Patients already using anakinra at baseline & $11(91.7)$ \\
Patients with history of other IL-1 blocking treatments at baseline & $3(25)$ \\
Anakinra dose at baseline, median (range), mg/kg/day & $1.7(1.1-2.5)$ \\
Anakinra dose during follow-up years 1:2:3:>3, median (range), mg/kg/day & $1.6: 1.6: 2.6: 1.4$ \\
& $(1.1-3.9: 1-4.4: 1.7-$ \\
Patients with AEs & $3.7: 1-3.2)$ \\
Patients with permanent discontinuation of anakinra & $1(8.3)$ \\
Patients with continuation of anakinra until the end of the study & $6(50)$ \\
Patients with temporary discontinuation of anakinra & $5(41.7)$ \\
Total duration of anakinra exposure, median (range), years & $1(8.3)$ \\
Any AE & $1.3(0.5-7)$ \\
Urinary tract infection & $7(100)$ \\
Upper respiratory tract infection & $1(14.3)$ \\
Tonsillitis & $1(14.3)$ \\
\end{tabular}

Results are shown as number (\%) unless stated otherwise.

Conclusion: The results of the present study confirm the safety profile of anakinra treatment in CAPS patients using the graduated pre-filled syringe. No new safety findings were identified.

Disclosure of Interests: Riccardo Papa: None declared, Gabriella Giancane: None declared, Helen J. Lachmann: None declared, Paul Brogan: None declared, Elizabeth Legger: None declared, Daniel Lindqvist Shareholder of: Sobi employee and holder of Sobi shares, Speakers bureau: Sobi employee and holder of Sobi shares, Paid instructor for: Sobi employee and holder of Sobi shares, Consultant of: Sobi employee and holder of Sobi shares, Grant/ research support from: Sobi employee and holder of Sobi shares, Employee of: Sobi employee and holder of Sobi shares, Susanna Cederholm Shareholder 